\title{
Do Patients with Asymptomatic Congenital Complete Heart Block Require a Pacemaker for Non-Cardiac Surgery?
}

\author{
Barry Swerdlow \\ Department of Anesthesia, Los Robles Regional Medical Center, Thousand Oaks, CA 91360, USA \\ Email: bswerdlow@verizon.net
}

How to cite this paper: Swerdlow, B. (2018) Do Patients with Asymptomatic Congenital Complete Heart Block Require a Pacemaker for Non-Cardiac Surgery? Open Journal of Anesthesiology, 8, 130-135. https://doi.org/10.4236/ojanes.2018.84014

Received: April 2, 2018

Accepted: April 27, 2018

Published: April 30, 2018

Copyright $\odot 2018$ by author and Scientific Research Publishing Inc. This work is licensed under the Creative Commons Attribution International License (CC BY 4.0).

http://creativecommons.org/licenses/by/4.0/

\section{(c) (i) Open Access}

\begin{abstract}
The appropriate preparation of the patient with asymptomatic congenital complete heart block (CCHB) and a narrow QRS complex for elective non-cardiac surgery is controversial. Prophylactic temporary pacemaker insertion is associated with well-defined risks, and less invasive techniques exist to treat transient, hemodynamically significant intraoperative bradyarrhythmias. The present case report details the performance of general anesthesia for arthroscopic knee surgery in an adult patient with this condition without a pacemaker. Documentation of preoperative chronotropic competence with isoproterenol may be of value in deciding whether to proceed without temporary pacing capability in this setting.
\end{abstract}

\section{Keywords}

Congenital Complete Heart Block, Pacemaker, Non-Cardiac Surgery

\section{Introduction}

CCHB results from electrical discontinuity between the atrioventricular (AV) node and the bundle of His or within the bundle of His, and can occur in the absence of other cardiac anomalies [1]. A significant percentage of patients with CCHB are asymptomatic, and the indications for permanent pacemaker insertion in the subset of this population with reasonable junctional rates, narrow QRS complexes, and normal ventricular function are controversial [2]. Despite the fact that there is limited literature to support the practice, temporary pacemakers are often inserted prophylactically for non-cardiac surgery in this setting [3]. However, such intervention can be associated with serious adverse events, including vascular complications, infection and arrhythmias. Hence, it is im- 
portant to define which patients will benefit from perioperative temporary pacing capability.

\section{Case Report}

A 56 year-old woman was admitted for arthroscopic-assisted anterior cruciate reconstruction with allograft. The patient had a history of asymptomatic complete heart block (CHB), dating from at least age 25 when she first noted a slow heart rate. Her only medication was levothyroxine. Blood pressure was 142/77 $\mathrm{mmHg}$ and heart rate 46 beats per minute (bpm). The patient's 12 lead ECG showed complete heart block with a narrow QRS complex and a normal QTc interval (Figure 1). Recent thyroid function studies were normal, and prior Lyme disease serology testing was negative.

Three years prior to admission, a 24-hour Holter study showed an average junctional rate of $43 \mathrm{bpm}$ (normal junctional rate $40-60 \mathrm{bpm}$ ), with a minimum rate of $30 \mathrm{bpm}$ and a single 2.6 second pause (normal $<3.0$ seconds). A stress echocardiogram documented no wall motion abnormalities, normal ventricular function, and normal valvular anatomy and function. The patient underwent an isoproterenol infusion according to a standard electrophysiologic protocol [4] two weeks prior to surgery that demonstrated a reasonable chronotropic response. During this study, the junctional rate increased from baseline (40 bpm) to a peak of $90 \mathrm{bpm}$ at an isoproterenol infusion rate of $6 \mathrm{ug} / \mathrm{min}$, with frequent ventricular premature beats but minimal change in blood pressure. Predicated on this data, preoperative cardiology consultation recommended against temporary pacemaker insertion.

After application of temporary pacing pads, general anesthesia was induced with propofol, a laryngeal mask airway was inserted, and anesthesia was maintained with sevoflurane (spontaneous respiration) and increments of fentanyl. The patient's junctional rate remained in the $40-50 \mathrm{bpm}$ range during the

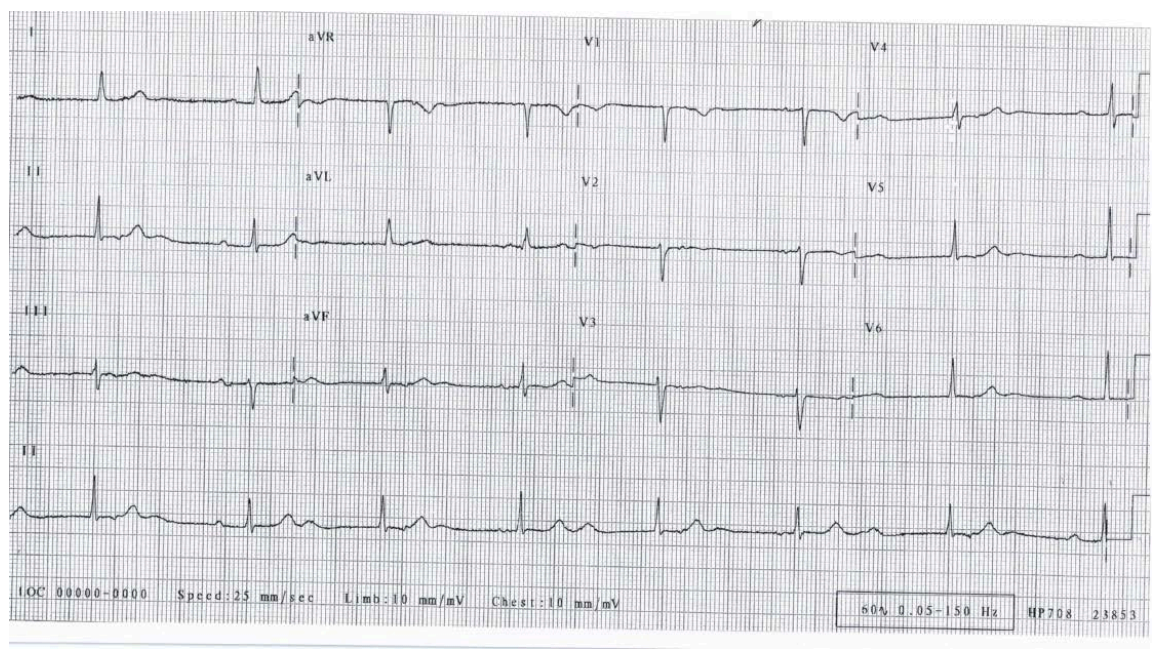

Figure 1. Preoperative 12 lead ECG showing a junctional rhythm at a rate of $48 \mathrm{bpm}$ with a QRS duration of $80 \mathrm{~ms}$, a QT interval of $461 \mathrm{~ms}$, and a QTc interval of $412 \mathrm{~ms}$. 
induction of anesthesia and the start of surgery. During curetting of the femoral condyle, her junctional rate dropped to $32 \mathrm{bpm}$, and an isoproterenol infusion was started at $4 \mathrm{mcg} / \mathrm{min}$ with an associated rise in junctional rate to $56 \mathrm{bpm}$. The patient's operative course was otherwise unremarkable, and the isoproterenol infusion was discontinued prior to extubation.

\section{Discussion}

Best practice indications for permanent pacemaker insertion in isolated CCHB are controversial [2]. Specifically, guidelines provide a Class IIb recommendation for pacing asymptomatic children or adolescents with an acceptable heart rate, a narrow QRS complex, and normal ventricular function [5]. Not only is the benefit of permanent pacemaker insertion uncertain in this population, but also adverse events from pacing in children are not uncommon [1] [2]. For this reason, anesthesiologists likely will see increasing numbers of asymptomatic, young patients with $\mathrm{CCHB}$ without permanent pacemakers who are scheduled for elective surgical procedures.

Historical indications for preoperative insertion of temporary pacing in asymptomatic patients with CCHB without evidence of ventricular dysfunction have included no chronotropic response to atropine and a QRS interval $>0.10$ seconds [6]. These criteria probably relate to the fact that lack of such pharmacologic response and the presence of a wide ECG complex are associated with conduction impairment at a level below the bifurcation of the His-bundle, rather than in the AV node, and thus are considered less stable under anesthesia. Both atropine and isoproterenol accelerate junctional automaticity, and therefore are likely to have reliable chronotropic effects in the setting of nodal (vs. infranodal) pathology. Use of an isoproterenol chronotropic response to define "chronotropic competence" differs from the usual methodology employed for this purpose, and a reasonable increase in junctional rate following administration of beta adrenergic pharmacology only fits the broad definition of the term [7]. Furthermore, these recommendations have not been studied in a controlled fashion, and an adequate clinical response to these drugs includes not only a reliable junctional chronotropic response, but also absence of hypotension and absence of isoproterenol-facilitated ventricular tachyarrhythmias [4]. In addition, when trying to decide which patients require temporary intraoperative pacing, multiple other factors require consideration such as the presence or absence of structural heart disease, the presence or absence of a prolonged QTc interval (likely a risk for bradycardia-induced Torsade de Pointes), the risk of intraoperative vagal stimulation, and the need for specific anesthetic pharmacology.

For example, certain operations are more likely to be associated with vagotonia and the potential for severe bradyarrhythmias. These include ocular surgeries, intra-abdominal procedures with peritoneal manipulation, laryngeal surgeries, and other procedures involving vagus nerve traction. Despite this fact, bowel resection has been uneventfully performed without pacemaker insertion in 
patients with asymptomatic CCHB with both a narrow QRS complex [6] and a wide QRS complex [8]. Likewise, co-morbidities (including narrow angle glaucoma where atropine is contraindicated) and a patient's ability to tolerate beta adrenergic stimulation (for example, coronary artery disease likely increases the risk of isoproterenol facilitated ventricular arrhythmias) and transient hemodynamic compromise need to be considered when deciding whether to place a prophylactic temporary pacemaker in this population.

Numerous drugs commonly employed during anesthesia can produce bradycardia and asystole, even in the absence of underlying conduction system disease. This phenomenon is commonly seen with acetylcholinesterase inhibitors and dexmedetomidine, but bradyarrhythmias have been reported with multiple other medications including propofol, opioids, and succinylcholine. Lifethreatening bradycardia in an infant with CCHB has been reported following halothane mask induction [9].

CCHB in asymptomatic pregnant patients with a narrow QRS complex and no underlying structural heart disease requires special consideration. Multiple cases have been reported of these patients undergoing uneventful delivery without pacemaker insertion [10]. However, prior to such a decision, defining chronotropic competence may be especially important given the dependence of cardiac output on heart rate at term. Isoproterenol is included in the algorithm for management of bradyarrhythmias in pregnancy and labor, and has been recommended for treatment of refractory symptomatic bradycardia in parturients with CCHB [11]. While the chronotropic effect of isoproterenol may be reduced in term pregnant women, a linear response does occur at higher doses [12]. Adverse effects of isoproterenol in pregnant women and fetuses are rare, and this drug has been used safely to treat CHB in fetuses [11]. Because neuraxial anesthesia delivered to the term parturient may impair sympathetic tone and require an increase in junctional rate to maintain blood pressure, appropriate pharmacologic response to atropine and/or isoproterenol is essential when employing such anesthesia if a temporary pacemaker insertion is not planned [10]. Prophylactic anticholinergic administration in this setting may be useful [13].

If a temporary pacemaker is not placed preoperatively, in addition to chronotropic pharmacology, a transcutaneous pacing system should be available intraoperatively. Preoperative placement of pacing pads is prudent. Problems associated with transcutaneous pacing include failure to capture (especially in patients with increased chest wall impedance), cutaneous burns, and ventricular arrhythmias [14].

Importantly, the etiology of $\mathrm{CHB}$ can be difficult to define when it initially presents in adulthood. Predicated on a thirty-year history of a slow junctional rate, a normal stress echocardiogram, and negative serology for Lyme borreliosis, CCHB was the likely diagnosis in our patient. An additional factor complicating management of these patients is that the first indication of a conduction disorder may occur intraoperatively when the patient is attached to the ECG 
monitor, especially given the relative infrequency of preoperative ECG determinations in young asymptomatic adults [15].

Current indications for temporary pacing of the asymptomatic adult patient with presumed $\mathrm{CCHB}$ and a narrow QRS complex requiring elective non-cardiac surgery are controversial. Important considerations in deciding whether such prophylactic intervention is prudent likely include the presence or absence of QTc prolongation, ventricular dysfunction or coronary artery disease, as well as the chronotropic response to atropine and/or isoproterenol and the nature of the non-cardiac surgery, specifically its propensity for vagotonia, blood loss, and hemodynamic compromise. The present case report illustrates the performance of an uneventful general anesthetic for a minimally invasive orthopedic procedure without temporary pacemaker insertion in a patient with probable CCHB, a narrow QRS, a normal QTc interval, normal ventricular function, and chronotropic competence. Future patients with similar or identical criteria can be satisfactorily managed without such intervention. Immediate availability of an isoproterenol infusion and transcutaneous pacing enhance safety in this setting.

\section{Conflicts of Interest}

The author has no conflicts of interest to declare.

\section{Informed Consent}

Informed consent for publication was obtained from the patient.

\section{References}

[1] Bordachar, P., Whinnett, Z., Ploux, S., Labrousse, L., Haissaguerre, M. and Thambo, J.B. (2013) Pathophysiology, Clinical Course, and Management of Congenital Complete Atrioventricular Block. Heart Rhythm, 10, 760-766. https://doi.org/10.1016/j.hrthm.2012.12.030

[2] Dolara, A. and Favilli, S. (2010) Controversies in the Therapy of Isolated Congenital Complete Heart Block. Journal of Cardiovascular Medicine, 11, 426-430. https://doi.org/10.2459/JCM.0b013e3283397801

[3] Csontos, C.S., Bogar, L. and Melczer, L. (2003) Temporary Pacemakers for Non-Cardiac Surgery. European Journal of Anaesthesiology, 20, 581-582. https://doi.org/10.1097/00003643-200307000-00014

[4] Freedman, R.A., Swerdlow, C.D., Echt, D.S., Winkle, R.A., Soderholm-Difatte, V. and Mason, J. (1984) Facilitation of Ventricular Tachyarrythmia Induction by Isoproterenol. American Journal of Cardiology, 54, 765-770. https://doi.org/10.1016/S0002-9149(84)80205-2

[5] Tracy, C.M., Epstein, A.E. Darbar, D., et al. (2013) 2012 ACCF/AHA/HRS Focused Update Incorporated into the ACCF/AHA/HRS 2008 Guidelines for Device-Based Therapy of Cardiac Rhythm Abnormalities. Journal of the American College of Cardiology, 61, e6-e75. https://doi.org/10.1016/j.jacc.2012.11.007

[6] Diaz, J.H. and Friesen, R.H. (1979) Anesthetic Management of Congenital Complete Heart Block in Childhood. Anesthesia \& Analgesia, 58, 334-336.

[7] Brubaker, P.H. and Kitzman, D.W. (2011) Chronotropic Incompetence: Causes, 
Consequences, and Management. Circulation, 123, 1010-1020. https://doi.org/10.1161/CIRCULATIONAHA.110.940577

[8] Linton, D.M. and Human, D.G. (1984) Peri-Operative Management and Administration of Anaesthesia in Children with Congenital Complete Heart Block. South African Medical Journal, 66, 875-876.

[9] Mohan, V.K., Naik, A.K., Bharti, N. and Shende, D. (2003) A Patient with Congenital Complete Heart Block Undergoing Multiple Exposures to General Anesthesia. Anaesthesia and Intensive Care, 31, 667-671.

[10] Sundararaman, L., Cohn, J.H. and Ranasinghe, J.S. (2016) Complete Heart Block in Pregnancy: Case Report, Analysis, and Review of Anesthetic Management. Journal of Clinical Anesthesia, 33, 58-61. https://doi.org/10.1016/j.jclinane.2016.01.021

[11] Herman, S.C. and Zhou, J. (2011) Isoproterenol Infusion for Treatment of Refractory Symptomatic Bradycardia in Parturients with Congenital Complete Heart Block. International Journal of Obstetric Anesthesia, 20, 361-363. https://doi.org/10.1016/j.ijoa.2011.05.003

[12] DeSimone, C.A., Leighton, B.L., Norris, M.C., Chayen, B. and Menduke, H. (1988) The Chronotropic Effect of Isoproterenol Is Reduced in Term Pregnant Women. Anesthesiology, 69, 626-628. https://doi.org/10.1097/00000542-198810000-00034

[13] Gupta, K., Balachander, H., Badhe, A. and Mohan, R.M. (2010) Low-Dose Spinal Anesthesia in Asymptomatic Complete Heart Block without Perioperative Pacing. Journal of Anesthesia, 24, 660-661. https://doi.org/10.1007/s00540-010-0938-x

[14] Kelly, J.S. and Royster, R.L. (1989) Noninvasive Transcutaneous Cardiac Pacing. Anesthesia \& Analgesia, 69, 229-238. https://doi.org/10.1213/00000539-198908000-00016

[15] O'Gara, J.P. and Edelman, J.D. (1981) Anesthesia and the Patient with Complete Congenital Heart Block. Anesthesia \& Analgesia, 60, 906-908. 